\title{
INTELLIGENT RESPONSIVE INDOOR SYSTEM (IRIS): A POTENTIAL SHOPLIFTER SECURITY ALERT SYSTEM
}

\author{
Mohamad Kuzahier Mohd Zahari \& Zarul Fitri Zaaba \\ School of Computer Sciences \\ Universiti Sains Malaysia, Malaysia
}

mkuzahier.ucom11@student.usm.my; zarulfitri@usm.my

\begin{abstract}
Shoplifting can occur at any time and any place. From the big mall to a small shop, many security measures have been put in place as prevention tools. Apparently, there are numbers of shoplifting prevention tools in the market such as the Closedcircuit Television (CCTV), Electronic Article Surveillance (EAS) and Future Attribute Screening Technology (FAST). However, the cost issues and the ease of use always become the main concerns for the shopkeepers. Therefore CCTV was widely accepted because of the ease and affordable price. Although the CCTV is their main preference, it can be noted that CCTV operates in a static way where it can only records and monitor the incidents. This paper highlights the conventional CCTV issues and proposes the Intelligent Responsive Indoor System (IRiS) the as security crime prevention tool that uses face detection, recognition and behavior analysis to detect potential shoplifting intentions. Six small shop owners were interviewed to understand their insights on the problems and the need to further enhance the current CCTV. In addition, detailed discussions were provided in relation to the development of IRiS. Therefore, it can be suggested that IRiS provides a significant foundation and promises to be a security prevention tool to improve the conventional functions of the CCTV.
\end{abstract}

Keywords: Security, face recognition, biometrics, security alerts, intelligent system, Human Computer Interaction. 


\section{INTRODUCTION}

Shoplifting is one of the most prevalent crimes. Shoplifters can enter the intended store at any time. However, it can be suggested that amongst the most vulnerable times are right after opening the shop, right before closing during shift changes and during associates, break time. Evidence shows that more than $\$ 13$ billion worth of goods are stolen from the retailers each year (Shoplifting Information Statistics 2015). It can be noted that the values were more than $\$ 35$ million per day. Shoplifting can occur anywhere on a busy street or even during peak hours. For instance, it was reported that 32 iphone gadgets were stolen and passed through the checkout point of the shop (Action News 2015). A store lost up to $\$ 13 \mathrm{~K}$ after a flash mob by a group of people where they performed and simultaneously pulled items from the racks (Gidman 2015). Other recent evidence in 2015 indicated that stores in the US reported a loss of $\$ 16.6$ billion due to shoplifting (Roney 2015). Many of these incidents of shoplifting were reported and had caused loss to the victims. In addition, they might have suffered traumatic experience and distress because of the incidents they experienced. Many tools have been used to prevent shoplifting. Even though there are many types of shoplifting prevention technology, the price of such technology sometimes can be very pricy. Thus, the shopkeepers face some difficulties to use such technology due to of budget constraints.

Shopkeepers can only pay attention to one or two customers at a time. They will be clueless of what the unattended customers are doing. In addition, they do not know which customers are potential shoplifters. Even though there are CCTVS in the shops, the shopkeepers will not be alerted of the CCTV footages when they are attending to customers. It is because the CCTV is used for monitoring and recording purposes only (i.e. static mode). Shopping complexes normally have a high standards of security precaution. This difference is due to the budget allocation given to shopping complexes to increase their security protection. Furthermore, they could afford have any of these security measures (i.e. security guards, automatic alarm systems and staff to monitor the CCTV at all times). For instance, they can afford to buy other shoplifting prevention tools such as electronic article surveillance (EAS) that is used to identify surveillance articles as they pass through a gated area in a shopping complex (HowStuffWorks, 2015). On the other hand, a small shop will rely on a small budget and the simplicity of the products. As the CCTV is widely used especially for a small shop, this paper investigated further how the CCTV functions can be improved and at the same time accommodate their budgets and be easy to use. Apparently, new technology has been introduced which is related to gender recognition, however, it is still new and in progress work (Arigbabu et al. 2015). 
The aim of this paper was to propose a crime prevention tool that overcomes shoplifting in small shops by utilising the surveillance camera using security image processing techniques and the biometrics approach on suspicious human behaviours to identify potential intruders or shoplifters before the incident happens. Once the incident happens, the system will alert the shopkeeper by displaying a warning on a computer (i.e. normally a screen which will be placed at the payment counter).

\section{RELATED STUDIES}

Security can be defined as protection from any adversaries with or without intention (Whitman \& Matford 2012). The fundamentals of information security cover three main elements which are confidentiality, integrity and availability. Computers become a medium of communication where many applications can be installed within it and cater to end-users, needs on a day-today basis. Having security tools in a shop or outlet is deemed to be a necessity. One possible reason for this is the threats that occur are so unpredictable. A shopping mall or a small shop needs to be maintained. Thus, manpower is needed to check and to refill the stock, and to observe customers coming in and going out, etc. When the shop size is huge or the shop lacks staff, it is crucial to look at who is coming in and going out of the shop so that no theft incidents occur in the shop. Observing customer actions or behaviour is crucial as there is a possibility that they might be committing.

Based on investigations, there are a few existing methods that have been used to solve the problem. Future Attribute Screening Technology (FAST) was created by the U.S. Department of Homeland Security. FAST is a system that predicts whether a person will or will not commit a crime (NewScientist, 2008). This system will monitor and track human body movements, voice pitch changes, eye movements, body heat changes, and breathing patterns (Cnet, 2011). Public places such as airports, malls and sports stadiums are the places for this system to be used or installed (Now Report, 2014).

Nowadays, most shopkeepers use Closed-circuit television (CCTV). CCTV is used to monitor and to record the surrounding activities to reduce the crime rate. Viewtech is presently the leading supplier for CCTV products and services in Malaysia. The latest technology used by Viewtech is Video Content Analysis (VCA) as a part of cloud computing services with SaaS (software as a service) (ViewTech, 2015). This CCTV can be applied in various market sectors such 
as airports, banks, commercial \& residential areas, education, government, industries, retail, etc.

The evolution of technology within this domain introduced Future Attribute Screening Technology (FAST) as a system to detect 'Pre-crime' in public places, but this system is not suitable to be used in small shops as the requirement of the screening process is too high. This system requires much hardware to perform the screening process such as a remote cardiovascular and respiratory sensor to measure the heart rate and respiration, a remote eye tracker, thermal cameras, a high resolution video camera and an audio system (Sutrop \& Laas-Mikko 2012). Based on the authors' knowledge, Malaysia does not have any specific 'Pre-crime' detection system for a specific shop but the standard practice by shopkeepers is to utilise CCTV. Generally, almost all small shops use CCTVs as their security measure. This is because the implementation cost of CCTV is quite cheap, roughly around RM230 (£35) for each CCTV (Pocket CCTV, 2015). However, CCTV does not have the capability to detect potential shoplifters and is unable to warn the shopkeeper of such theft occurrences. Besides CCTV, Electronic Article Surveillance (EAS) is also one of the shoplifting prevention tools that widely used. EAS tags are used together with the merchandise and will be removed at the counter when the payment has been made (Agon, 2015). The EAS antenna normally will be placed at the exits of the store and an alarm will be sounded when it senses an active EAS tag on the merchandise. Nowadays, many shops are already using this to prevent shoplifting.

Table 1

Comparison of Shoplifting Prevention Systems

\begin{tabular}{|c|c|c|c|c|}
\hline Criteria & FAST & CCTV & EAS & IRiS \\
\hline Category & Application & Application & Non-Application & Application \\
\hline Hardware & $\begin{array}{c}\text { Camera, Thermal } \\
\text { Camera, remote } \\
\text { eye tracker, audio } \\
\text { system }\end{array}$ & $\begin{array}{l}\text { Video camera, } \\
\text { Computer }\end{array}$ & $\begin{array}{c}\text { Electronic antenna } \\
\text { deactivator, and } \\
\text { electronic tag }\end{array}$ & $\begin{array}{l}\text { Video camera, } \\
\text { Computer, } \\
\text { alarm }\end{array}$ \\
\hline Face recognition & Yes & No & No & Yes \\
\hline Alarm & Yes & No & Yes & Yes \\
\hline \multirow[t]{2}{*}{ Price } & High & Low & Medium & Low \\
\hline & $\begin{array}{c}\text { Airport, } \\
\text { Shopping mall, }\end{array}$ & $\begin{array}{l}\text { Shopping mall, } \\
\text { Small shop }\end{array}$ & Shopping mall & Small shop \\
\hline
\end{tabular}


Table 1 shows the comparison of the shoplifting prevention systems. It can be noted that only FAST and EAS use a lot of hardware in compared to the CCTV and IRiS. When a system uses a lot of hardware, the price of the system will potentially become more expensive. FAST and EAS are suitable to be implemented in large areas such as shopping malls. On the other hand, CCTV is widely used in most shops, whether they are big or small in size. In terms of the face recognition function, FAST utilises this technology with some other functions. IRiS is expected to use a technology similar to FAST but with less hardware. In addition, the price can be significantly reduced as less hardware is used.

This paper highlights IRiS as one possible means of the security prevention system which is currently being developed. Intelligent Responsive Response System (IRiS) is a system that detects potential shoplifters in small shops. This system will help the shop owners to stay alert of their customers to prevent shoplifting. IRiS utilises CCTVs and desktops/laptops as its main hardware. Hence, it will be suitable for small shops to use this system. IRiS will be integrated with the CCTV system to perform face recognition and the human pose recognition process in order to calculate the percentage of potential shoplifters. Then IRiS will generate an alarm to warn the shopkeepers.

\section{Preliminary Study}

In the preliminary study, interviews were conducted to further ascertain the usage of the tools of potential criminal detection within small shops. Thus, six shop owners around Sungai Dua, Penang, Malaysia were randomly chosen and interviewed. This area has many small shops such as stationary shops, small restaurants, convenience store etc. The interviews provided useful insights of the security prevention technologies owners used in their shops. The following questions (open ended) were asked: as 1 . Do you use any security tool or system to improve your shop's security measure? 2. If yes, what type of tool or system do you use? 3. Do you know how CCTV works as a security system? 4. Does CCTV help to reduce the rate of shoplifting in your shop? 5. Are you alert to any display on the CCTV? 6. Does shoplifting happen in your shop even with the use of CCTV? 7. Is the cost to implement the security measure (i.e. CCTV, etc.) important? 8. If there is a new security system which helps you to detect and give out an alarm when suspicious individuals are in your shop, would you use it?

The results derived from Table 2 indicate that all small shops were using a security system and CCTV was their main choice. They chose CCTV, because the cost to implement the CCTV was relatively low and CCTV was easy to 
maintain. With CCTV as their security prevention system, shops 1, 3, 4 and 6 claimed that the rate of shoplifting in their shop was reduced. Shops 2 and 5 were unsure of the change because the owners claimed that the number of shoplifters who entered their shops was too small. Shoplifters would act when they realize the shopkeepers are not paying attention to them. Based on question 5, only shop 1 and 5 stay alerted with any display on the CCTV because they had an extra worker in their shops. When the shopkeepers see any suspicious customers, they would ask their worker to approach the customer. On the other hand, the other shopkeepers claimed they were not really alert with the CCTV. If a suspicious customer was in the shop (i.e. by instinct), they approached the customer by themselves. This situation (i.e. lack of staff/ focus) would provide a huge opportunity to other potential shoplifters in the shop to get a chance to commit theft.

Table 2

Interview Results

\begin{tabular}{ccccccc}
\hline $\begin{array}{c}\text { Shop/ } \\
\text { Question }\end{array}$ & Shop 1 & Shop 2 & Shop 3 & Shop 4 & Shop 5 & Shop 6 \\
\hline 1 & Yes & Yes & Yes & Yes & Yes & Yes \\
2 & CCTV & CCTV & CCTV & CCTV & CCTV & CCTV \\
3 & Yes & Yes & Yes & Yes & Yes & Yes \\
4 & Yes & Not sure & Yes & Yes & Not sure & Yes \\
5 & Yes & Sometimes & ometimes & ometimes & Yes & ometimes \\
6 & Yes & Yes & Yes & Yes & Yes & Yes \\
7 & Yes & Depends on & Yes & Depends & Yes & Yes \\
8 & $\begin{array}{c}\text { Depend on } \\
\text { cost and } \\
\text { ease of sure }\end{array}$ & Possibly & Possibly & Yes & Possibly & Possibly \\
& & & & & \\
\hline
\end{tabular}

All shops claimed that shoplifting still happens in their shops even when they have CCTVs. They only realize the theft when the recorded video is played back on the CCTV. In terms of implementing a security measure, most of the interviewees agreed that costing was important. They needed to consider this factor before implementing any security measures in their shops. For the final question, most shopkeepers answered they possibly would use the enhanced CCTV provided they were an affordable and easy to use. 


\section{System Requirements}

IRiS is designed to help the shopkeeper to stay alert with the CCTV. An alarm will be activated when the value in the system exceeds the threshold. Iris involves the Web camera (i.e. act as a CCTV), a computer (i.e. laptop or desktop) and the IriS software. IRiS has similar capabilities as other available products with regards to notification, alarm and video recording. On the other hand, it differs and provides more functions in relation to face detection, face recognition and behavioral analysis. The main capabilities of IRiS are:

- able to recognize the face of the customer that enters the shop,

- able to upload the pictures of the potential shoplifters,

- $\quad$ able to track the same customer with more than one CCTV, and

- generate an alarm when the threshold is exceeded (i.e. potential shoplifters).

\section{PROPOSED SOLUTION}

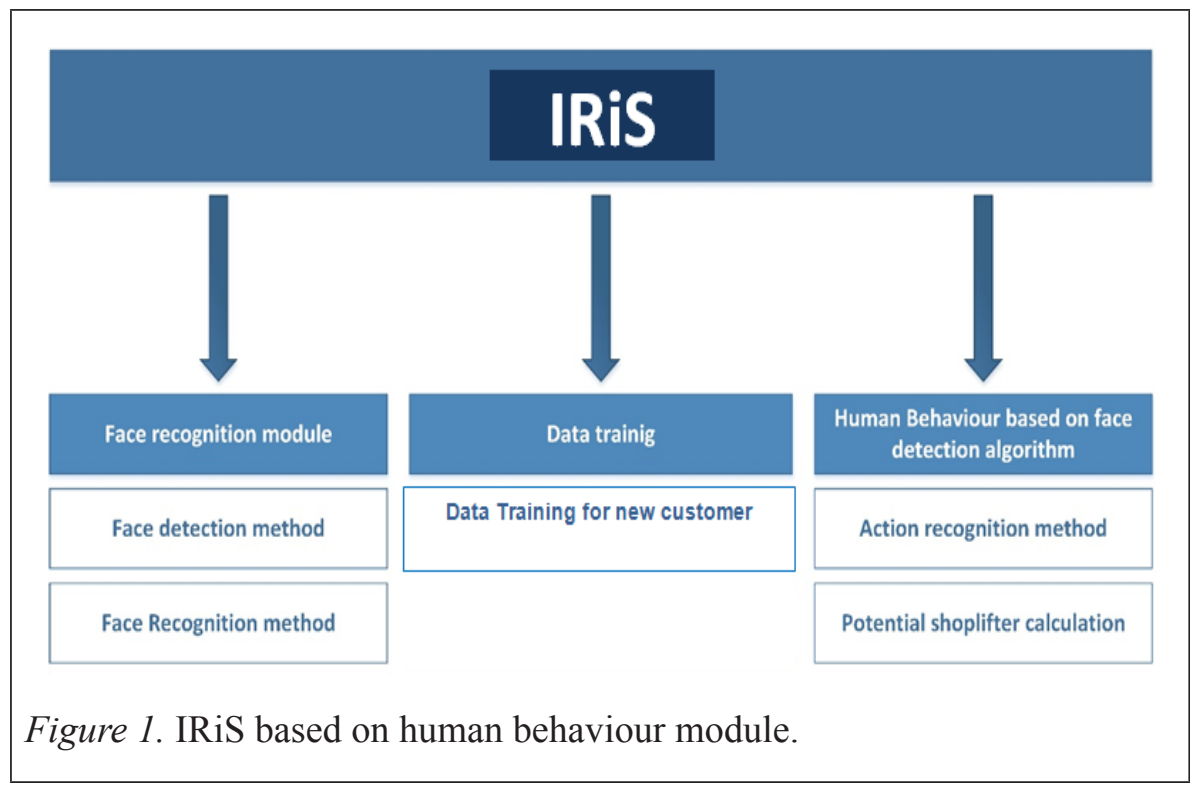

Based on the interview results, most of the shops were still using the CCTV as their prevention tool or security measure. Shoplifting still occured from time to time and the CCTV did not have the capability to detect or recognise potential shoplifters. Thus, the shopkeepers were not aware of any potential shoplifting activities. This paper introduces IRiS to bridge the gap in these 
issues. IRiS is an application which is be able to calculate the percentage of one who has the potential to be a shoplifter (i.e. based on behaviour analysis face gesture movements) and once the percentage exceeds a certain threshold, the shopkeeper will go and ask the potential shoplifter if he/she needs any help (i.e. distract them from commiting any shoplifting).

The Intelligent Responsive Indoor System (IRiS) is a system that utilises video cameras and computers. Basically of IRiS is to be used as a crime prevention tool that utilises face detection, recognition and behaviour analysis to detect the potential shoplifting intentions. IRiS also will help the shopkeepers to be alerted to the CCTV and to their surroundings. IRiS consist sof three main modules (Figure 1); face recognition module, data training module and human behaviour module that uses face detection and face recognition.

\section{System Design and Implementation}

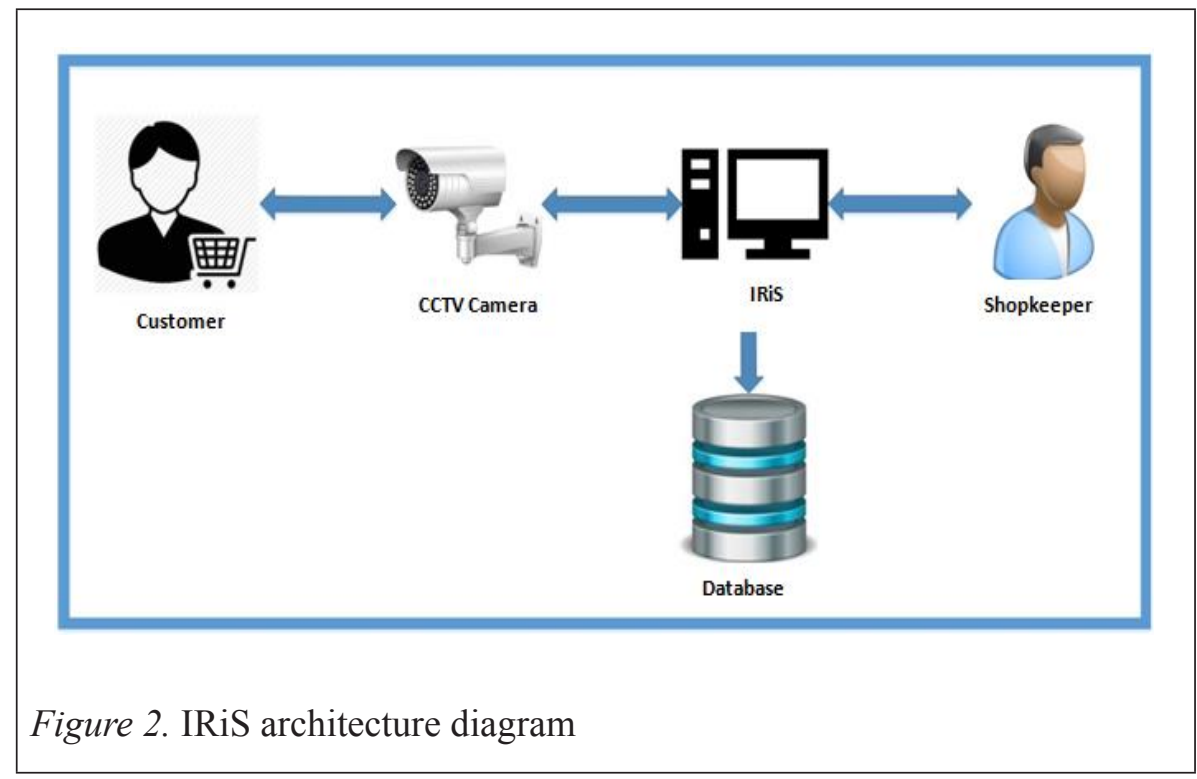

The hardware used in IRiS is depicted in Figure 2. The customer will be captured via the CCTV and later will be saved in the database. The IRiS will process the data and do the calculation based on the identified algorithm (i.e. face recognition algorithm; Eigenfaces, Fisherfaces and Local Binary Pattern) and upon the successful detection of potential shoplifters, an alarm will be triggered to indicate the shopkeeper or the staff needs to approach the customer. 


\section{Modules in IRiS}

The face recognition module consists of two sub-modules which are the face detection method and the face recognition method. On the other hand, the data training module consists of one sub-module which is customer data training. The human behaviour module consists of two sub-modules which are the action recognition method and the calculation module. The IRiS will calculate the customer's potential percentage to be a shoplifter by recognizing the customer's faces. When the customer's potential percentage to be a shoplifter reaches the threshold that has been set, an alarm will be activated (i.e. at the payment counter/on the screen). Then, the shopkeeper or the staff can approach the customer in a polite manner to ask them if help is needed (i.e. to prevent shoplifting).

\section{Face Detection}

The first function of this system is the face detection function. This function detects and captures the customer's face (e.g. CCTV 1 captures). The face detection function will be activated when a customer enters the shop and the data will be saved in the database which later will be used in the face recognition phase.

The face detection function starts with loading the face detector as shown in Figure 3.Once the image is loaded, the image is read. The following line code converts the captured frame into a greyscale image.

gray_frame $=$ currentFrame. Convert $\langle$ Gray, Byte $\rangle()$;

After that, the grayscale image undergoes the histogram equalization. Then, it detects the face in the grayscale image by using the face classifier. We use the "Haar" cascade classifiers as shown in the following codes.

public HaarCascade Face = new HaarCascade(Application. StartupPath + "/Cascades/haarcascade_frontalface_default. $x m($ ');

Based on the given codes, "Face" is the variable for the "Haar" cascade and it is used in the detection parameter. The line code (Face, 1.2, 3, Emgu. CV.CVEnUm. HAAR_DETECTION_TYPE. DO_CANNY_PRUNING, new Size $(25,25)$ ) is the parameter to use the "Haar" cascade classifier and also the parameter for scaling the detected faces (i.e. complete line of codes as depicted below) 
MCvAvgComp[][]facesDetected=gray_frame.DetectHaarCascade(Face, 1.2, 3,E mgu.CV.CvEnum.

HAAR_DETECTION_TYPE.DO_CANNY_PRUNING, new Size $(25,25))$;

After the system detects the faces by using the "Haar" cascade classifier, the rectangle is detected outside the detection face as shown in Figure 4 (i.e. as the final result).

result=currentFrame.Copy (face_found.rect). Convert $\langle$ Gray, byte>().

Resize(100,100,Emgu.CV.CVEnum.INTER.CV_INTER_CUBIC);

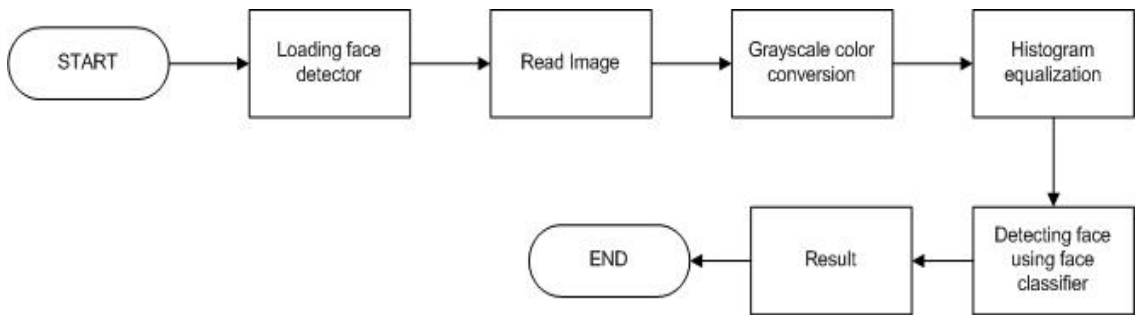

Figure 3. Face detection flow chart.

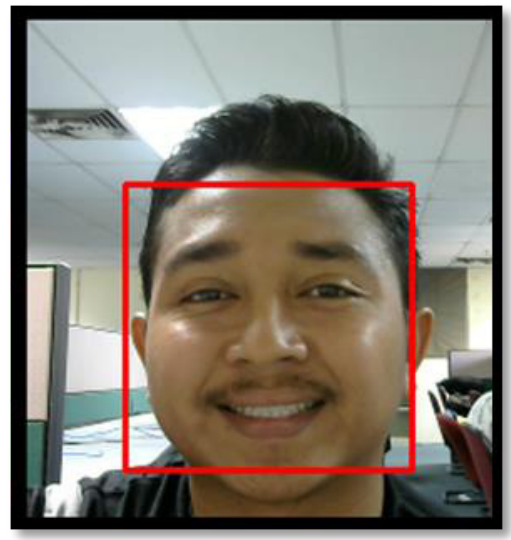

Figure 4. Face detection results. 


\section{Face Training}

The face training flowchart is shown in Figure 5. It is compulsory before the face recognition function is able to recognise the customer's face. The face training consists of two parts, which are face detection and face training. The face detection process is similar to that discussed in section 3.2. At the beginning of the face training it utilizes the face detection algorithm to capture the face.

if (! save_training_data(face_PICBX.Image)) MessageBox.Show ("Error", "Error in saving file info. Training data not saved", Message BoxButtons.OK, MessageBoxIcon.Error);

The code above is to train and to save the image into the database. "(!save training_data(face_PICBX.image))" means, by using the function "save_ training_data", the system takes the image in the picture box named "face PICBX.image" to train and save into the database which is the in XML database format. After that, the face undergo as the smoothing process to ensure the smoothness of the image. Then comes the process of elliptical mask comes in place where the image is created with one or more masks for each layer in a composition before it stores the pre-process face. If the process is completed, the training image is saved with the details of name, date and time. These attributes are used in the XML file accordingly as depicted in the codes below.

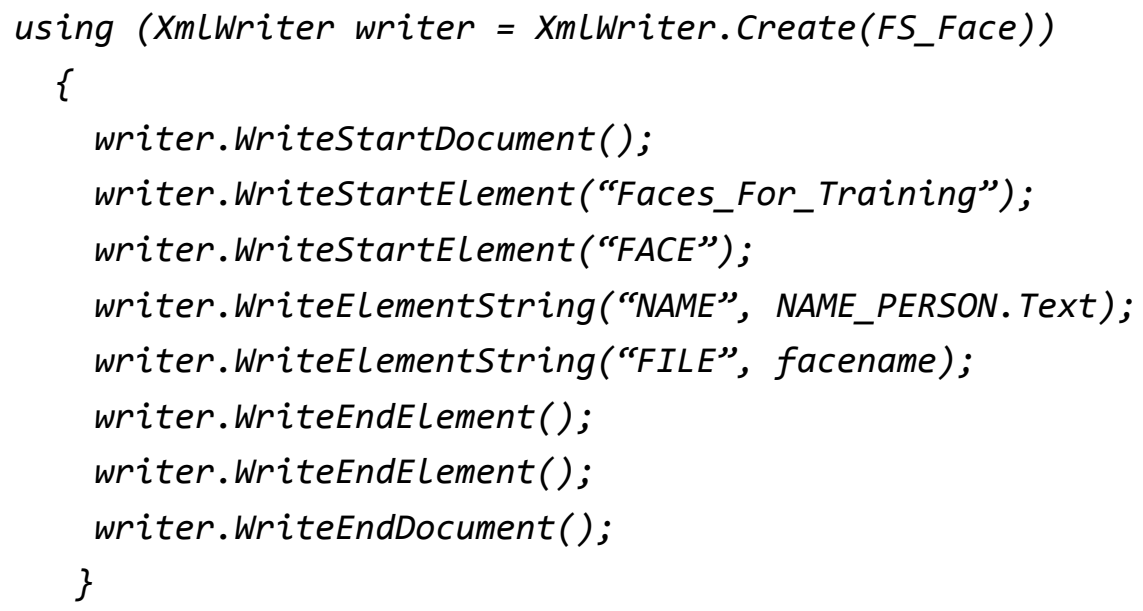

After the completion of the training stage, the training face in the XML file will be saved as shown in Figure 6. 


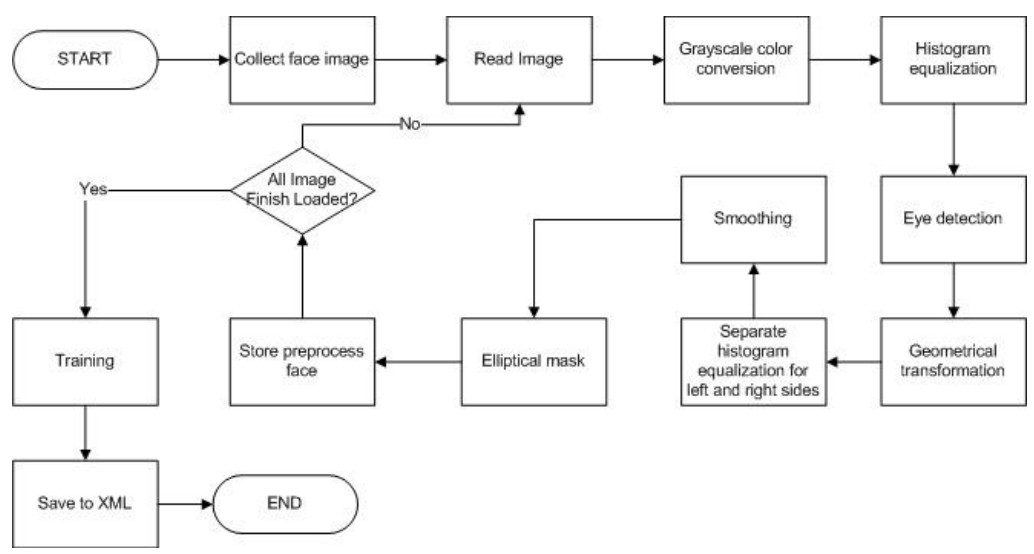

Figure 5. Face training flow chart.

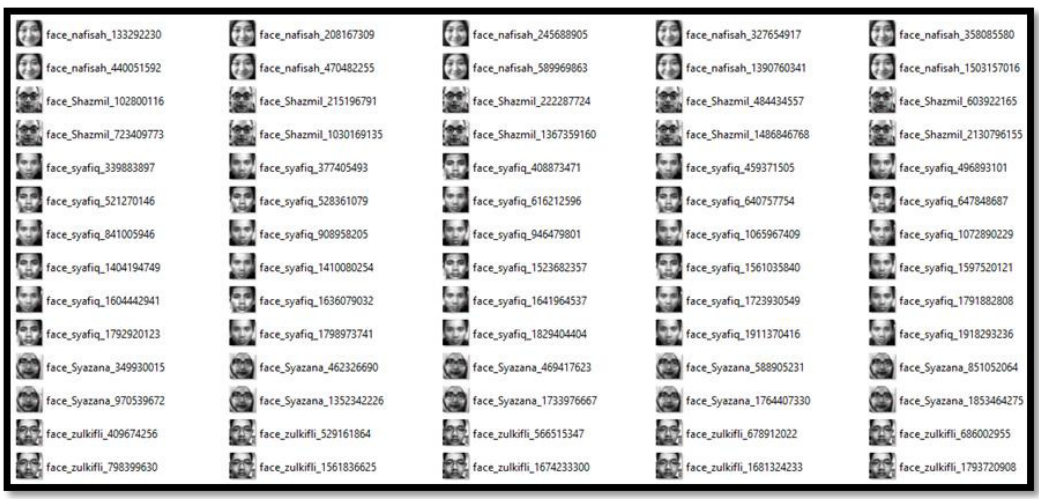

Figure 6. XML database.
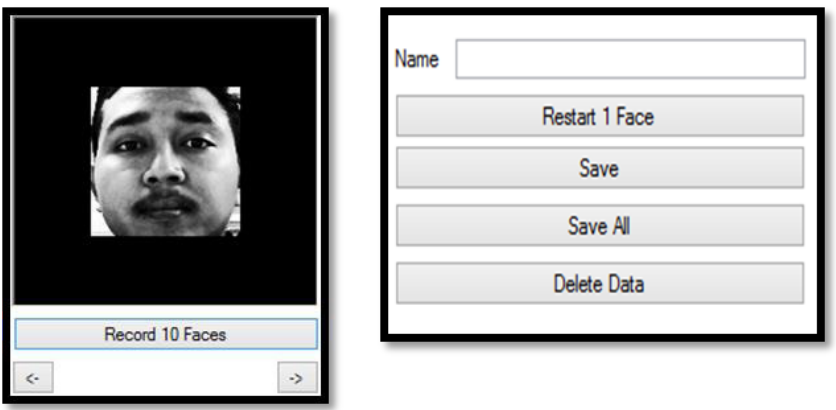

Figure 7. Results of training faces. 
Figure 7 shows the process of face training. Later the data will be saved in a specified folder to be used as a XML file.

\section{Face Recognition}

The face recognition module in IRiS plays an important role to recognise every customer who enters the shop. Generally, in small shops there will be at least two CCTVs (e.g. CCTV 1 to capture the first image of the customer and CCTV 2 to track the customer and monitor the customer's behaviour). This module will help IRiS to keep track of the same customers in the different CCTVs. The face recognition process considers the customer as a new customer if the customer's data does not match the image in the database (i.e. face detection phase). Thus, each new customer's image will be captured and trained (e.g. CCTV 2 will track and monitor the customer's behaviour).

The customer's behaviour will be monitored by CCTVs in the areas that have been specified by the shopkeeper. A customer's behaviour (i.e. gesture or movement) will contribute to the percentage calculation. The IRiS only focuses on a customer's movement, which is how often the customer looks at the CCTV. The IRiS chooses these behaviours because of two main reasons. Firstly, a potential shoplifter is often jittery or nervous before they commit any crime. Looking at the CCTV many times indicates that they are trying to cover up their actions or pretending to act normally. Secondly, most shoplifters are well aware of the surroundings where the current CCTVs are located and the shopkeepers' whereabouts before commiting the crime (Gifford, 2015). Finally, IRiS will calculate the total percentage. Therefore, if the customer looks at the CCTV many times, it will increase the threshold that has been set earlier. If the value exceeds the threshold, it will trigger an alarm at the counter which indicates that the shopkeeper or a member of the staff should go and politely ask if the customer needs any help.

The face recognition flowchart is shown in Figure 8. Similar to the face training phase, face recognition also consists of two parts; face detection and face recognition. After the face is detected in the face detection part, it undergoes the eye detection process. Then it will separate the histogram equalization for left and right sides. This is the process that involves image processing to provide a contrast adjustment so that the image can be better distributed. After that the face will undergo the smoothing stage to ensure the smoothness of the image. Then comes the process of the elliptical mask where the image is created with one or more masks for each layer in a composition before it stores the pre-process face. After that, the face will be matched with the training 
face in the database by using "eigenfaces", "fisherfaces", and "Local Binary Pattern (LBPH)". The "FaceRecognizer" is a global constructor that is able to use all three classifiers together. Each of these classifiers are discussed further in the next section.

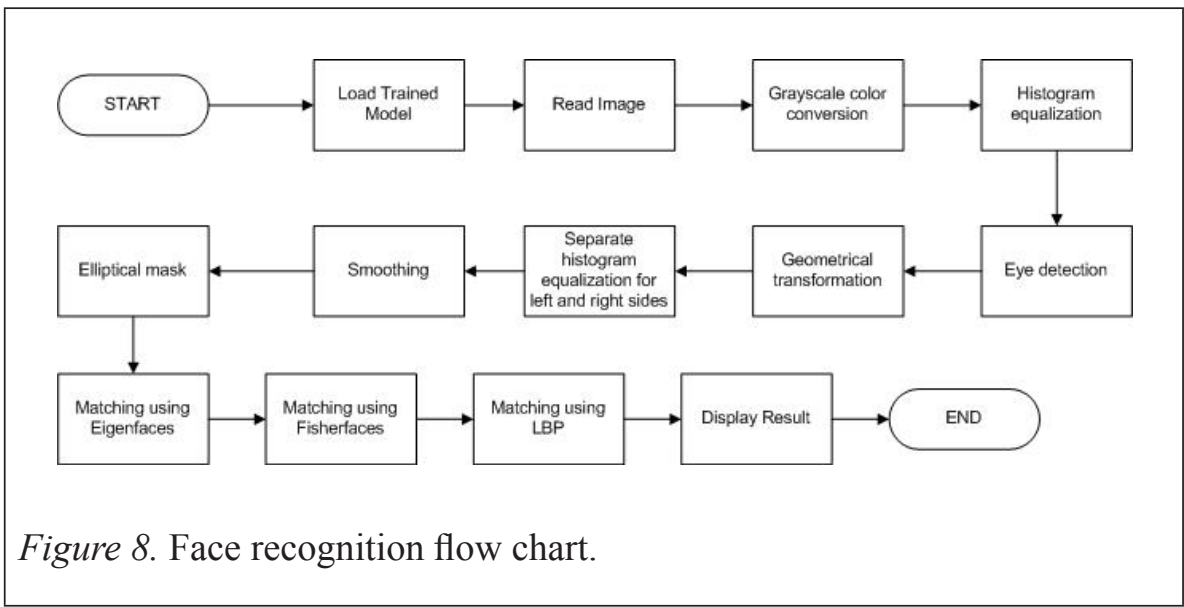

\section{Face Recognition Methods}

"Eigenface"s is a face recognition method developed by Matthew Turk and Alex Pentland based on Principal Component Analysis (PCA) (Turk \& Pentland, 1991). PCA is also known as the "Karhunen-Loeve" method which was developed by Kirby and Sirovich in 1988 (Kirby \& Sirovich, 1990). This method aims to reduce the feature dimension based in the database. This method uses PCA to reduce the feature dimension. The main concept of this method is encoding the extracted face feature information and comparing with the encodings in the database. Figure 9 shows an example of a standard "eigenfaces". "Eigenfaces" uses PCA to search the most discriminating features between a set of face images. The face space was created based on the best variation between the faces. Each face image is projected into the faces space after subtracting the mean face. The euclidean distance between two faces is computed to compare the faces (Tsalakanido et al., 2003).

"Fisherfaces" is an enhancement of "eigenfaces". "Fisherfaces" method applies the Linear Discriminant Analysis (LDA) for face recognition. LDA is a statistical method used in pattern recognition to calculate a linear combination of features that classify objects. The "fisherfaces" method uses LDA to reduce dimensions and simplify the classifiers in the reduced face subspace. Compared to the "eigenfaces" method, this method is more complex but has better performance under constraints like illumination and facial pose. 


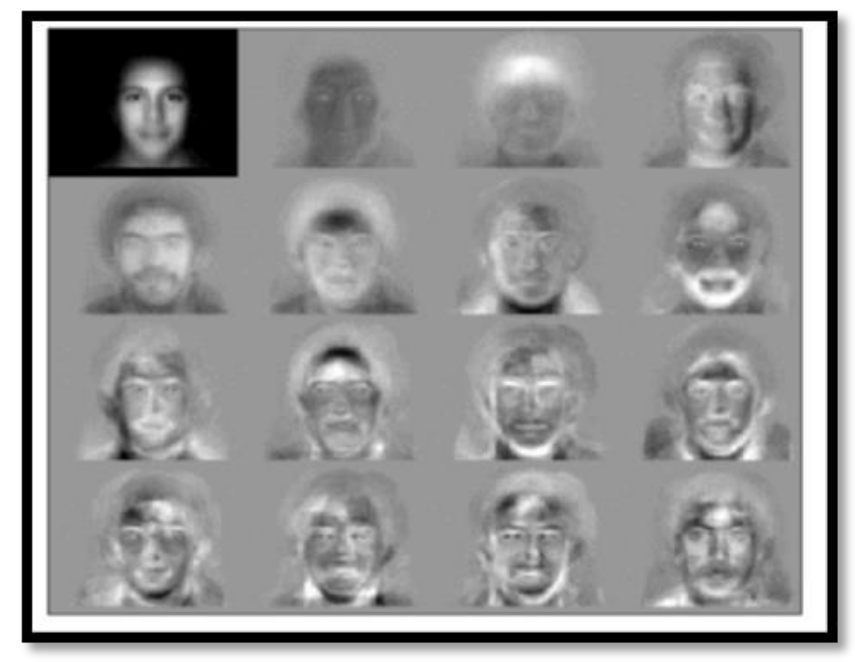

Figure 9. Standard Eigenfaces (Turk \& Pentland, 1991).

The concept of Local Binary Patterns (LBP) is different from PCA and LDA. LBP describes only local features of an object instead of the high dimensional vector of the whole image (Ahonen et al., 2004). The features extracted will have a low dimensionality implicitly. The basic idea of the Local Binary Patterns is to process the local structure in an image by comparing each pixel with the adjacent pixels. The intensity of the center pixel will be the threshold to compare with the adjacent pixel. If the center pixel has a greater intensity, then it will denote the adjacent pixel with 1 and 0 . A binary number for each pixel will be generated.

An experiment was done on to compare the "eigenfaces" and "fisherfaces" methods. The both experiment used the database from the Yale Center (Figure 10) to compare the methods with variations in facial expression, eye wear, and lighting. The result of the experiment shows that the "fisherfaces" method has a far lower error rate compared to the "eigenfaces" method (Belhumeur et al., 1997).

EMGU CV is a cross platform .Net wrapper to the "OpenCV" image processing library. It allows the "OpenCV" functions to be called from .NET. The "FaceRecognizer" is a global constructor that allows "Eigen" and "Fisher" classifiers to be used together. The class combines common method calls between the classifiers. The constructor for each classifier type is as follows: 


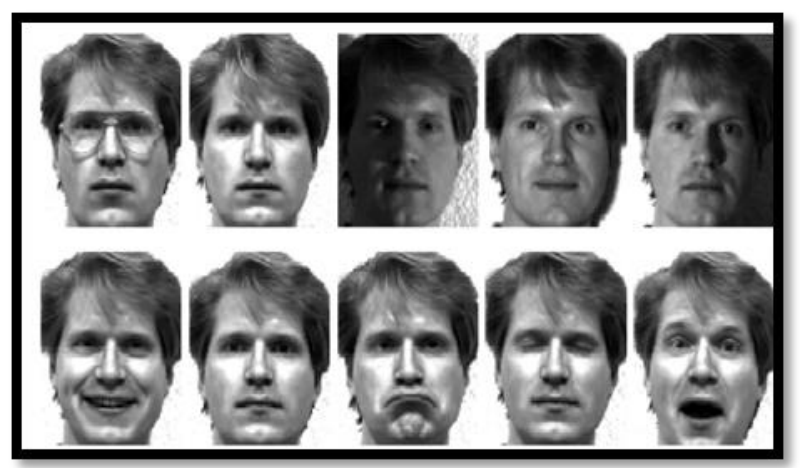

Figure 10. A sample of the Yale database, an individual under 10 different conditions (Belhumeur et al., 1997)

\section{The Eigen Classifier}

FaceRecognizer recognizer $=$ new EigenFaceRecognizer(80, double . PositiveInfinity);

The "Eigen recognizer" takes two variables. The first variable is the number of components kept for the Principal Component Analysis. There is no specific rule on how many components should be kept for good reconstruction capabilities. "OpenCV" documentation suggests that keeping 80 components should almost always be sufficient. The second variable is designed to be a prediction threshold; this variable contains the bug as any value above this is considered as an unknown. For the Fisher and LBHP, this is how unknowns are classified, however, with the Eigen recognizer the return distance must be used to provide the own test for the unknowns. In the Eigen recognizer the larger the value returned the closer to a match (Johnson 2014).

\section{The Fisher Classifier}

FaceRecognizer recognizer $=$ new FisherFaceRecognizer $(\theta, 3500)$; $/ / 4000$

The "Fisher" recognizer takes two variables like the "Eigen" constructor. The first variable is the number of components that keep the Linear Discriminant Analysis with the "Fisherfaces" criterion. If left at the default (0) and set to a value less than 0 , or greater than the number of the training inputs, it will set to the correct number (chosen training inputs - 1) automatically. The second variable is the threshold value for the unknowns. If the results of the 
Eigen distance is above this value, the Predict( ) method will return a -1 value indicating an unknown. This method works and the threshold is set to a default of 3500 . However, if the value in the constructor is changed, the recognizer will need to be retrained (Johnson, 2014).

Based on the literature, Table 3 shows the summary of the comparison between "Eigenfaces" and "Fisherfaces". The result implies that the "Fisherfaces" method has better face recognition performance than "Eigenfaces" under constraints of facial pose and illumination. For the complexity of the algorithm, "Fisherfaces" algorithm is more complex compared to the "Eigenfaces" algorithm. Thus, the performance under various poses of "fisherfaces" algorithm is good. However, "Fisherfaces" algorithm has low sensitivity to lighting compared to "Eigenfaces". Based on these comparisons, IRiS will utilize both algorithms because the CCTV will need to be placed at a certain location which has significant impacts in terms of shop size, light exposure, etc. Thus, both algorithms are capable at least in one of those situations/ requirements.

Table 3

Comparison Based on Complexity, Facial Pose and Lighting

\begin{tabular}{lcc}
\hline & Eigenfaces & Fisherfaces \\
\hline Complexity & Simple & More Complex \\
$\begin{array}{l}\text { Performance under various } \\
\text { poses }\end{array}$ & Low & Good \\
Sensitivity to lighting & High & Low \\
\hline
\end{tabular}

\section{DISCUSSION}

IRiS is developed based on the significant number of shoplifting crimes reported (e.g. theft, privacy breach etc). Although the incidentsoccur in big malls or small shops, they indicate that possible actions can be taken to improve the current security measures that have been put in place. This paper proposed IRiS as a security or crime prevention tool that utilises face detection, recognition and behavior analysis to detect potential shoplifting intentions. The focus of this proposed solution is based on small shops which need to be more cost savvy and the tools should be easy to use. The conventional approach of the CCTV only captures or records the incidents without any notification for further actions. IRiS is introduced as a new form of prevention 
where the CCTV will be able to detect and generate an alarm for an informed decision to the shopkeeper or staff (i.e. politely approach the customer or potential shoplifter). It should be noted that IRiS is not a problem-solving tool, but it is more of a mechanism to reduce the actual risk before it occurs. Three algorithms of recognition system have been identified; Eigenfaces algorithm, Fisherfaces algorithm and LBPH algorithm. They will be used accordingly (i.e. various facial poses, complexity and lighting sensitivity, space etc). The preliminary study interviewed six shop owners to understand the insights on the problems and the need of enhancement of current CCTV implementation. Two the issues that were highlighted were the cost and the ease of use factors. IRiS can be viewed as being able to counterbalance the problems.

The IRiS has a notification feature for a shopkeeper to keep alert with the CCTV (i.e. via alarm notification at the counter). The existing shoplifting prevention system like EAS is not suitable for a small shop. It is because the EAS consume large number of hardware and the cost to implement it is rather expensive. Thus, CCTV was an affordable choice as their security measure. Although CCTV was widely used, it has some weaknesses. CCTV seems to be static and it only records the incidents. CCTV also does not have any notification feature to warn the shopkeeper or the staff members. Furthermore, the shopkeeper will only realize the particular incident only after he/she views the recorded tape of the CCTV which would be to late to avoid the incident from happening.

\section{CONCLUSION}

In conclusion, conventional CCTV has been widely used by most shops, especially in Malaysia and even in other parts of the world. The main reasons for choosing this tool are of the cost and ease of use. However, there are still some significant issues and challenges that need to be taken care of. As the CCTV is operating in a static mode (i.e. records only), it can be enhanced to make it more useful and meaningful as a usable security tool. As suggested, CCTV can be operated utilizing face detection, recognition and behavior analysis which provide more convincing results. The authors are currently in the final phase of developing the prototype of this proposed solution. The results of this development are convincing. The development of IRiS faces a significant number of challenges such as high complexity of image processing / detection phases, the accuracy issues and false alarm rates which are expected to be improved from time to time. IRiS has room for improvement in future 
works such as the integration of emotion, movement and gesture in order to refine the identification of potential shoplifters. IRiS has a promising future as a security intelligent system on a small scales which is able to provide better security for all.

\section{REFERENCES}

Action News. (2015). Shoplifter goes through checkout with 32 stolen iphones. Retrieved from http://6abc.com/news/shoplifter-goes-throughcheckout-with-32-stolen-iphones/1043391/

Ahonen, T., Hadid, A., \& Pietikainen, M. (2004). Face recognition with Local Binary Pattern. Lecturer Notes in Computer Science, 3021, 469-481.

Arigbabu, O. A., Syed Ahmad, S. M., Wan Adnan, W. A., Yussof, S., \& Mahmood, S. (2015). Soft biometric: Gender recognition from unconstrained face images using local feature descriptor. Journal of ICT, 14, 111-122.

Agon. (2015). Electronic article surveillance. Retrieved 8 December 2015 from http://www.agon-systems.com/electronic-article-surveillance/

Belhumeur, P. N., Hespanha, J. P., \& Kriegman, D. J. (1997). Eigenfaces vs. Fisherfaces: Recognition using class specific linear projection. IEEE Transaction on Pattern Analysis and Machine Intelligence, 19 (7), 771-720.

Cnet. (2011). Homeland security moves forward with 'Pre-crime' detection - CNET. Retrieved 8 December 2015 http://www.cnet.com/news/ homeland-security-moves-forward-with-pre-crime-detection/

Gidman, J. (2015). Flash Mob steals $\$ 13 \mathrm{~K}$ from Tony Georgetown store. Retrieved 10 December 2015 from http://www.newser.com/story/ 216644/flash-mob-steals-13k-from-tony-georgetown-store.html

Gifford, P. (2015). How to spot potential shoplifter. Retrieved 10 December 2015 from http://www.ehow.com/how_4596958_spot-potential-shoplifter. html.

HowStuffWorks. (2015). Electronic article surveillance. Retrieved 4 December 2015 from http://electronics.howstuffworks.com/everydaytech/anti-shoplifting-device2.htm 
Johnson, C. (2014). EMGU multiple face recognition using PCA and parallel optimization. EMGU multiple face recognition using PCA and parallel optimization-CodeProject. Retrieved 10 December 2015 from www. codeproject.com.

Kirby, M., \& Sirovich, L. (1990). Application of the Karhunen-Loeve procedure for the characterization of human faces. IEEE Transactions on Pattern Analysis and Machine Intelligence, 12(1), 103-108.

NewScientist. (2008). Short sharp science: Pre-crime detector shows promise. Retrieved 9 December 2015 from https://www.newscientist.com/blogs/ shortsharpscience/2008/09/precrime-detector-is-showing-p.html

Nwo Report. (2014). Future attributes screening technology. Retrieved 7 December 2015 from http://nworeport.me/tag/future-attributescreening-technology

Pocket CCTV. (2015). Malaysia CCTV camera price. Retrieved 10 December 2015 from http://www.pocketcctv.com.my/Camera.html

Roney, L. (2015). US retrial worker stole $\$ 16.6$ billion from employers this year. Retrieved 10 December 2015 from http://www.newser.com/ story/216206/us-retail-workers-stole-166b-from-employers-this-year. html

Shoplifting prevention. (2014). Shoplifting information and statistics. Retrieved 3 December 2015 from https://www.shopliftingprevention. org/whatnaspoffers/NRC/PublicEducStats.htm

Sutrop, M., \& Laas-Mikko, K. (2012). From identity verification to behavior prediction: Ethical implication of second generation biometrics. Review of Policy Research, 29, 21-36.

Tsalakanidou, F., Tzovaras , D. , \& Strintzi, M. G. (2003). Use of depth and colour eigenfaces for face recognition. Pattern Recognition Letters, 24, 1427-1435.

Turk M., \& Pentland, A. (1991). Face recognition using eigenfaces. Proceedings of IEEE Computer Vision and Pattern Recognition. Maui, Hawaii, 586-591. 
ViewTech. (2015). IP CCTV Malaysia |IP CCTV Supplier Malaysia |IP CCTV Manufacturer Malaysia. Retrieved 5 December 2015 from at: http://www.viewtech.com.my/Company-Profile/

Whitman M. E., \& Mattord H. J. (2012). Principle of information security. Course Technology, Cengage Learning.ISBN: 9781111138233. 